\title{
Research advances in the targeted therapy and immunotherapy of Wilms tumor: a narrative review
}

\author{
Bo Hong^, Rui Dong^ ${ }^{\wedge}$ \\ Department of Pediatric Surgery, Children's Hospital of Fudan University, Shanghai Key Laboratory of Birth Defect, and Key Laboratory of \\ Neonatal Disease, Ministry of Health, Shanghai, China \\ Contributions: (I) Conception and design: B Hong; (II) Administrative support: R Dong; (III) Provision of study materials or patients: R Dong; (IV) \\ Collection and assembly of data: B Hong; (V) Data analysis and interpretation: B Hong; (VI) Manuscript writing: All authors; (VII) Final approval of \\ manuscript: All authors. \\ Correspondence to: Rui Dong. Department of Pediatric Surgery, Children's Hospital of Fudan University, Shanghai Key Laboratory of Birth Defect, \\ and Key Laboratory of Neonatal Disease, Ministry of Health, 399 Wan Yuan Road, Shanghai 201102, China. Email: rdong@fudan.edu.cn.
}

\begin{abstract}
Wilms tumor is the most common pediatric abdominal solid tumor, and its treatment has been a focus of research. For now, the 5 -year survival rate of children with Wilms tumor is about $90 \%$. It is difficult to make further progress simply by the improvement of the existing treatments (multi-modal therapy). Therefore, targeted therapy and immunotherapy which have high accuracy and few side effects began to be considered for the treatment of Wilms tumor. At present, though targeted therapy and immunotherapy are rarely used in the treatment of Wilms tumor except in clinical trials, there are dozens of clinical trials research them around the world. The sites in targeted therapy research are mainly focused on insulinlike growth factor 2 (IGF2) pathway, anti-angiogenesis, phosphoinositide 3-kinase (PI3K)/protein kinase B (AKT) signaling pathway, and some miRNAs, etc. And there are three types of study in Wilms tumor immunotherapy, which are inhibition of the COX-2 pathway, chimeric antigen receptor (CAR)- $\mathrm{T}$ cell therapy, and multi-tumor associated antigen (TAA)-specific cytotoxic T lymphocytes (CTL) therapy. Among them, the phase I clinical trial of multi-TAA-specific CTL (MTAA-CTL) therapy has been completed, and the results are very satisfactory. In this narrative review, we review the basic research and relevant clinical research on targeted therapy and immunotherapy for Wilms tumor.
\end{abstract}

Keywords: Wilms tumor; targeted therapy; immunotherapy

Submitted Nov 22, 2020. Accepted for publication Jan 08, 2021.

doi: $10.21037 /$ tcr-20-3302

View this article at: http://dx.doi.org/10.21037/tcr-20-3302

\section{Introduction}

Wilms tumor, also known as nephroblastoma, is the most common renal tumor in children, accounting for more than $90 \%$ of pediatric renal tumors (1). Most children with Wilms tumor went to hospital because their parents find palpable abdominal lumps. In addition, some children also have symptoms such as hematuria, fever, urinary tract infection, varicocele, hypertension or hypotension, anemia, etc. (2) In recent years, with the development of multi-modal therapy based on the combination of surgery, chemotherapy and radiotherapy, the 5 -year survival rate of children with Wilms tumor is about $90 \%$ (1-3). However, studies have shown that the recurrence rate of Wilms tumor is about $15 \%$ and the long-term survival rate of recurrent Wilms tumor is only $50 \%$ (4). Wilms tumor with "anaplastic" histology has a low five-year survival rate, especially the "diffuse anaplastic" histology in stage IV $(<30 \%)(5)$. It is also difficult to make a breakthrough in the multi-modal therapy of Wilms tumor. Therefore, targeted

^ ORCID: Bo Hong, 0000-0001-6964-7273; Rui Dong, 0000-0001-9645-4768. 
therapy and immunotherapy which have high accuracy and few side effects have been considered for the treatment of Wilms tumor. So far, although targeted therapy and immunotherapy are rarely used in the treatment of Wilms tumor, there are dozens of clinical trials research them around the world (from https://clinicaltrials.gov/). We present the following article in accordance with the Narrative Review reporting checklist (available at http:// dx.doi.org/10.21037/tcr-20-3302).

\section{Targeted therapy}

Anti-tumor targeted therapies focus on the specific characteristics of tumor cells, which are essential for the initiation and maintenance of tumors. Because of their specificity, targeted therapies generally cause fewer side effects than chemotherapy and radiotherapy (6). In terms of action mechanism, the current research on targeted drugs is mainly focused on the following four aspects.

\section{Insulin-like growth factor 2 (IGF2) pathway inbibition}

The $I G F 2$ signaling pathway is closely related to the development of Wilms tumor, and the combination of upregulated $I G F 2$ gene expression and Wilms tumor 1 (WT1) gene ablation leads to Wilms tumor (7). There are two proven reasons for the overexpression of IGF2. First, the loss of DIS $3 L 2$ gene function leads to the transcriptional activation of IGF2/H19 site in primary nephron progenitor cells, which further leads to the overexpression of IGF2 in $W T$ (8). Second, the mutation of some miRNAs (such as $m i R-16$ and $m i R-34)$ in Wilms tumor leads to inhibition of the $I G F 2$ regulator PLAG1, and overexpression of PLAG1 enhances the expression levels of IGF2 (9). For the $I G F 2$ pathway, $I G F 1 R$, the $I G F 2$ receptor, is currently considered the most feasible therapeutic target due to its overexpression and its role in the occurrence and growth of cancer. The $I G F 2$ signaling pathway is closely associated to the development of Wilms tumor, and $I G F 2$ receptor $I G F 1 R$ is one of the most feasible therapeutic targets. The use of the gene translation initiation site with complementary antisense oligonucleotide targeting IGF1R $m R N A$ to halt $I G F 1 R$ expression, small molecule inhibitors, or monoclonal antibody blocking $I G F 1 R$ instead of the interaction between ligands can inhibit the proliferation of Wilms tumor cell lines. IGF1R inhibitors BMS-754807 and NVP-AEW541 are currently in experimental stage. BMS-754807 is a competitive ATP small molecule that can significantly inhibit tumor growth when used in a xenotransplantation mice model of Wilms tumor. The application of NVPAEW541 in Wilms tumor can simultaneously inhibit the downstream mitogen-activated protein kinase signaling pathway of IGF2 and the expression of CCNA2 and $C C N B 1$, which can inhibit tumor growth. Recent studies have shown that $I G F 1 R$ acts as a tyrosine kinase in the $I G F$ pathway and has kinase-independent activity, suggesting that it may be more effective to combine targeted therapy with monoclonal antibodies and small molecule inhibitors targeting IGF1R (10).

\section{Anti-angiogenesis therapy}

The rapid growth and development of tumors require intensive angiogenesis and rapid proliferation, while nontumor sites have slow or no proliferation of blood vessels. Therefore, angiogenesis has an important role in the development and metastasis of tumors and inhibiting this process will obviously prevent the development and spread of tumor tissues.

The vascular endothelial growth factor (VEGF)/ vascular endothelial growth factor receptor (VEGFR) pathway is the most commonly targeted pathway in antiangiogenic therapy. $V E G F$ is a known factor that induces angiogenesis. Anti-angiogenic therapy targeting $V E G F R$ is widely used in cancer therapy. Among them, $V E G F-A$ is the most thoroughly studied factor inducing endothelial cell proliferation and angiogenesis (11). VEGF- $A$ expression in the serum and tissues of Wilms tumor patients is related to poor prognosis, which lays a theoretical foundation for antiangiogenesis therapy (12). $V E G F-A$ regulates angiogenesis and vascular permeability by activating two receptors, VEGFR-1 and VEGFR-2 (13). Apatinib is a small molecule anti-angiogenic agent that selectively binds to and inhibits the kinase activity of $V E G F R-2$, thereby reducing $V E G F-$ mediated migration and proliferation of tumor endothelial cells and thus reducing tumor microvascular density and inhibiting the growth of Wilms tumor. Currently, bevacizumab, AZD2171, and other VEGF/VEGFR pathway inhibitors are on the market or are in clinical trials (12).

There may also be other targeted pathways for antiangiogenesis therapy. VEGF is overexpressed in Wilms tumor, but the expressed subtype of $V E G F$ is determined by the shear mode of the precursor of $V E G F$. Wilms tumor suppressor 1 (WT1) activates serine/arginine-rich proteinspecific splicing factor kinase (SRPK1) and indirect serine/ arginine-rich splicing factor 1 (SRSF1) activity in tumor 
vascular endothelial cells, thereby inducing the expression of the pro-angiogenic subtype of $V E G F$. Specific knockout of $W T 1$ can reduce the expression of SRPK1 and SRSF1 endothelial cells, thereby inducing the expression of $V E G F$ anti-angiogenic subtype VEGF120 (11). Therefore, WT1 could be a potential target in anti-angiogenesis therapy.

Antiangiogenic therapy, however, can lead to rashes, elevated lipases, anorexia, blood clots, and pneumothorax. Pneumothorax is the most serious complication of antiangiogenic therapy in children with Wilms tumor and can even be life-threatening (14). Therefore, the risk of pneumothorax should be evaluated during antiangiogenic therapy.

\section{Inbibition of the phosphoinositide 3-kinase (PI3K)/protein kinase B (AKT) signaling patbway}

The PI3K/AKT signaling pathway has a critical role in tumor development. This pathway can be activated by cell surface receptors (such as tyrosine kinase receptors), G-protein coupled receptors, and oncogenes expression product (such as Ras superfamily), and activated PI3K can catalyze the conversion of phosphatidylinositol 4, 5-diphosphate to phosphatidylinositol 3,4,5-triphosphate, thereby activating $A K T$. AKT signaling is transmitted to a variety of downstream effectors, including mammalian target rapamycin complex 1 (mTORC1), to regulate various cellular processes. When activated abnormally, this pathway may be involved in the regulation of proliferation, apoptosis, migration, and invasion of Wilms tumor cells (15).

In terms of tumor therapy, a variety of inhibitors such as buparlisib have been developed against $P I 3 K$ and its subtypes, $A K T, m T O R$ and other pathway proteins. As the most advanced inhibitor targeting PI $3 K$ and its subtypes, buparlisib is applied to human tumor cell lines with $P I 3 K$ pathway changes, which can inhibit the proliferation of tumor cells and promote the apoptosis of tumor cells. In mice transplanted with PIK3CA mutations, buparlisib can significantly inhibit tumor growth and even cause tumor regression. However, buparlisib is more toxic, so studies on it have focused on reducing toxicity. Currently, only mTORC1 allosteric inhibitors such as everolimus and temsirolimus are approved for clinical use (15).

Inhibition of this pathway can not only directly inhibit the pathway proteins, but also target the regulatory factors of the pathway proteins and the upstream activating genes of the pathway. The regulatory factors that have been proven to be potential target sites are PTEN/PTEN pseudogene
(PTENP1) (16), and the activation gene is KRAS (17). PTEN is a negative regulator of $P I 3 K$. By dephosphorylation of phosphatidylinositol 3,4,5-triphosphate at the $\mathrm{d} 3$ position of the inositol ring, phosphatidylinositol 4,5-diphosphate is formed to directly antagonize the effect of PI3K. The loss or inactivation of PTEN leads to overactivation of RTK/PI3K/ $A K T$ signal transduction, which leads to tumorigenesis. PTENP1 transcription products can competitively bind to PTEN mRNA to block its expressed miRNA, and inhibition of PTENP1 transcription has been experimentally demonstrated to reduce PTEN expression (16). RAS is a proto-oncogene. In the Wilms tumor cells, RAS mutations can activate the $P I 3 K / A K T$ signaling pathway together with $\beta$-catenin, thus promoting the proliferation, migration and invasion of the Wilms tumor cells, as well as tumor growth and lung metastasis (17).

\section{A new approach to targeted therapy: targeted miRNA and miRNA as a targeted drug}

miRNA is a small single-stranded non-coding RNA that can regulate gene expression at the post-transcriptional level by binding to mRNA 3'UTR to inhibit protein translation or promote mRNA degradation. miRNA expression profiles of different subtypes of Wilms tumor cell lines and normal renal tissue cell lines were constructed and analyzed by miRNA microarray technology and quantitative reversetranscription PCR. The results showed that miRNA expression in the two cell types was significantly different (18-21). Subsequent studies have found that miRNAs can regulate the proliferation, migration, and apoptosis of Wilms tumor cells by regulating the expression of pathway proteins in cell signaling pathways, and thus play an important role in the occurrence and development of Wilms tumor. Therefore, miRNAs can be used as potential target sites and even as targeted drugs for targeted therapy of Wilms tumor (22-28).

Currently, seven miRNAs have been confirmed to have an important role in the occurrence and development of Wilms tumor, including miR-891b (22), miR-21 (23), $m i R-19 b$ (24), miR-483-3p (25), miR-140-5p (26), miR613 (27), and $m i R-572$ (28). Their expression imbalance and regulated cell signaling channels in Wilms tumor cells are shown in Table 1. miR-21, $m i R-19 b, m i R-483-3 p$, and $m i R-891 b$ can positively regulate the $P I 3 K / A K T$ pathway, promote the proliferation, migration, and invasion of Wilms tumor cells, and inhibit their apoptosis, but their regulatory mechanisms may be different (22). PTEN mRNA is the 
Table 1 Imbalance in miRNA expression in Wilms tumor and its regulated cell signaling channels

\begin{tabular}{lcc}
\hline miRNAs & Expression & Signaling channels \\
\hline miR-891b & Up & NF-kB, PI3K/AKT \\
miR-21 & Up & PI3K/AKT \\
miR-483-3p & Up & PI3K/AKT \\
miR-19b & Up & PI3K/AKT \\
miR-572 & Up & CDH1 \\
miR-140-5p & Down & TGFBRI/SMAD2, 3 \\
miR-613 & Down & FRS2 \\
\hline
\end{tabular}

direct target of $m i R-21, m i R-19 b$, and $m i R-483-3 p$, which can promote the $P I 3 K / A K T$ pathway by downregulating $P T E N$ expression at the post-transcriptional level (23). The mechanism of the positive regulation of $m i R-891 b$ is still unclear and further studies are needed (22). MiR-613 and $m i R-140-5 p$ are two tumor-suppressing miRNAs that are downregulated in the expression of Wilms tumor cells (26). However, miR-613 can directly target FRS2 mRNA and downregulate the expression of $F G F 2$ at the posttranscriptional level to inhibit the proliferation, migration, and invasion of tumor cells (27). MiR-140$5 p$ can directly target $T G F B R 1$ and $I G F 1 R$ mRNA, downregulate $T G F B R 1$ and $I G F-1 R$ expression after transcription, inhibit the $I G F 1 R / A K T$ and TGFBR1/SMAD2/3 pathways, and further inhibit the occurrence and development of Wilms tumor (26). Therefore, $m i R-613$ and $m i R-140-5 p$ can not only be used as therapeutic target sites, but also be directly used as targeted drugs, which need to be verified by drug experiments.

Unfortunately, due to the lack of research on the role of miRNAs in the occurrence and development of Wilms tumor, no drugs targeting this site have been investigated in clinical trials. However, research has found that salidroside can be adjusted by $m i R-891 b$ to inhibit the $N F-\kappa B$ and $P I 3 K / A K T / m T O R$ signaling pathways, thereby inhibiting the proliferation and metastasis of Wilms tumor cells, and thus several studies have attempted to elucidate the pharmacological action of rhodiola glucoside as a potential therapy (22).

In addition to the target sites mentioned above, there are also some potential target sites, such as RECK gene (29), KCNQ1OT1 gene (30), nerve cell adhesion molecule (31), hypoxia-inducing factor-1 (32), LINC00473 (33), etc. These potential targeting sites provide some new directions for future drug development.

\section{Immunotherapy}

Anti-tumor immunotherapy is a treatment method to control and eliminate tumor cells by restarting and maintaining the tumor-immune cycle and restoring or even enhancing the normal anti-tumor immune response of the body (34). Both tumor immunotherapy and targeted therapy can target a specific protein, but immunotherapy can stimulate and enhance the immune capacity of the body through this target. Currently, two main methods are applied in immunotherapy against Wilms tumor: inhibition of the cyclooxygenase-2 $(C O X-2)$ pathway and adoptive cellular immunotherapy.

\section{Inbibition of the COX-2 pathway}

$C O X-2$, one of the two isoenzymes of prostaglandin, is robustly expressed in the inflammatory microenvironment of Wilms tumor (35). A model of Wilms tumor in mice was previously established. WT1 ablation and IGF2 upregulation were used to stimulate the Wilms tumor microenvironment in the mouse model, and the expression of $C O X-2$ pathway components in the mouse model was observed and their roles were analyzed. Immunohistochemistry showed that $C O X-2$ pathway components, such as $C O X-$ 2 , hypoxia-inducible factor- $1 \alpha$, and mitogen-activated protein kinase, were highly expressed in the mouse models. Flow cytometry analysis revealed increased infiltration of immunosuppressive immune cells such as regulatory dendritic cells and regulatory $\mathrm{T}$ cells in the tumor. Through real-time quantitative PCR studies, immunosuppressive cytokines such as interleukin-10 and transforming growth factor- $\beta$ were found to be upregulated in the mouse model, and the expression of chemokines such as chemokine-CC motif-receptor-5 and C-X-C motif chemokine receptor 4 that induce the infiltration of these immunosuppressive immune cells was also upregulated. It can be inferred from the above evidence that the $C O X-2$ pathway has an important function in the production and transport of immunosuppressive immune cells, which participate in the formation of the tumor immune microenvironment of Wilms tumor and play an important role in tumor immune escape. Therefore, targeting $C O X-2$ can inhibit tumor immune escape (36). 


\section{Chimeric antigen receptor (CAR)-T cell therapy}

CAR-T cell therapy refers to the use of gene modification technology (CRISPR/Cas9) to transfer genetic material with specific antigen recognition domains and $\mathrm{T}$ cell activation signals into $\mathrm{T}$ cells $(\mathrm{CD} 8+\mathrm{T}$ cells), so that $\mathrm{T}$ cells can be directly combined with specific antigens on the surface of tumor cells to be activated to treat tumors $(37,38)$. In the past decades, CAR has undergone four generations of structure. In the first generation of CAR, there is only the activation signal from the $C D 3 \zeta$ chain. When the $\mathrm{scF} v$ recognizes the tumor associated antigen (TAA), the signal is transferred into the $\mathrm{T}$ cell, and the $C D 3 \zeta$ signal triggers the activation of $\mathrm{T}$ cells. In the second and third generation of CAR, one or two costimulatory signals were added to respectively to enhance the proliferation of $\mathrm{T}$ cells, tumor cell killing, and cytokine secretion (IL-2, TNF, $I F N-$, etc.) (39-41). The fourth generation of CAR-T cell contains an transcriptional response element of nuclear factor of activated $\mathrm{T}$ cells, which enables CAR-T cells to secrete specific cytokines (mainly $I L-12$ at present) in the tumor area, thus modifying the tumor microenvironment, recruiting and activating other immune cells for immune response $(41,42)$. However, overactivity of CAR-T cells is likely to cause cytokine release syndrome, and the duration of CAR-T cells in vivo is relatively short (43). Wu et al. found that $C D 3 \varepsilon$ can recruit inhibitory signal molecules $C s k$, through its immunoreceptor tyrosine-based activation motif signal, leading to impaired signaling of the CD3 $/$ Zap70/Plcy 1 axis and subsequent reduction of cytokines; and recruit $p 85$ through its basic residue rich sequence signal motif, which promotes $A K T$ signal transduction and CAR-T persistence; the fifth generation CAR-T has begun to form (44).

There are some specific targets such as CD19, CD22 and $C D 123$ (only in cancer cells but not in normal cells) in blood cancer, these targets can lead CAR-T cells to find and destroy cancer cells (45). Therefore, CAR-T cell therapy is effective in blood cancer. At present, CD19 CAR-T cell therapy has been approved by FDA to be used in the treatment of acute lymphoblastic leukemia and diffuse large B-cell lymphoma (46). However, there is no such targets in Wilms tumor $(47,48)$, and cytolytic CD8+ T cells are confined to intravascular circulation (49). Therefore, the application of CAR-T cell therapy in Wilms tumor is still in clinical trials. The existing clinical trials are as follows:

Glypican-3 (GPC3) is the first proposed target for CAR-T cell therapy of Wilms tumor. GPC3 is a heparan sulfate proteoglycan on the cell surface, which is anchored to the cytoplasmic membrane by glycosylphosphatidylinositol and can interact with a variety of regulatory proteins important for cell growth and differentiation, including Wnt, Hedgehog and fibroblast growth factor (50). The transcriptional and proteomic expression of GPC3 in Wilms tumor was significantly higher than that in adult renal tumor and normal renal tissue. Combined with the results of three studies evaluating the expression of $G P C 3$ in nephroblastoma, 50/87 (58\%) cases showed GPC3 expression, and somatic tumor mutations of GPC3 were even found in some Wilms tumors $(51,52)$. Since the expression of GPC3 in Wilms tumors is not lower than in hepatoblastoma $(131 / 135,97 \%)$ (51), the existing GPC3 CAR-T cell immunotherapy for pediatric solid tumors (ClinicalTrials.gov Identifier: NCT04377932) only recruits hepatoblastoma patients, while Wilms tumor patients are included in the scope of recruitment in the future.

Epidermal growth factor receptor $(E G F R)$ is another target that may be suitable for CAR-T cell therapy of Wilms tumor, which binds to epidermal growth factor to induce activation of receptor-related tyrosine kinases and ultimately promotes DNA synthesis, proliferation and differentiation of target cells (53). Shuai-Jun Dong, master of Zhengzhou University, performed HE staining on 35 cases of Wilms tumor, 14 cases of peritumoral renal tissue and 8 cases of normal renal tissue. It was found that the positive rate of $E G F R$ was $62.86 \%$ in nephroblastoma and $28.57 \%$ in peritumoral renal tissue, and no expression in 8 cases of normal renal tissue (54). Given the high specificity of EGFR in Wilms tumor, clinical trial of EGFR806 CART cell therapy for recurrent/refractory solid tumors in children and young people (ClinicalTrials.gov Identifier: NCT03618381) recruited Wilms tumor patients at the initial stage, and the first phase of the trial is expected to end in June, 2021.

The positive rate of the above targets in Wilms tumor is about $60 \%$, which is not enough to label all tumor tissues, so the application of CAR-T cell therapy in Wilms tumor patients progressed slowly. A recent breakthrough by the Majzner team at Stanford University offers hope to CAR-T cell therapy for Wilms tumor. The Majzner team screened samples from 388 children's tumors and found that 325 (84\%) were positive for $B 7-H 3,70 \%$ of which showed high intensity staining for $2+$ or $3+$. All the 12 Wilms tumor specimens were stained with high intensity $(2+$ or $3+)(55)$. On this basis, the Majzner team developed a new generation of B7-H3 CAR-T cell therapy for solid tumors. This 
special CAR-T cell therapy is regarded as one of the most promising therapies. Recently, a clinical trial has begun to recruit Wilms tumor patients (ClinicalTrials.gov Identifier: NCT04483778).

\section{Multi-TAA specific cytotoxic T lymphocytes (MTAA-CTL)}

MTAA-CTL is one of the fifth generation CTL cell therapy, which is characterized by the directional expansion of MHC-restricted CD8+ NK-T cells while ensuring the expansion of non-MHC-restricted CTL cells, so that the ratio of MHC-restricted CD8+ CTL cells in cellular products can reach $60-70 \%$. The two kinds of cells work together to improve the efficiency of killing tumor cells. Compared with CAR-T cell therapy, which can only target single surface antigen, MTAA-CTL cell therapy can extract dendritic cells from peripheral blood monocytes and coculture with immune cells after being processed with multiple tumor antigens (adding related antigens according to the tumor of the patient: one is the antigen extracted from the patient's blood, the other is the broad-spectrum antigen peculiar to the cancer). So, acquired CTLs can target a variety of surface antigens. The obtained DC-CTL cells were infused intravenously, which could effectively and specifically recognize and kill tumor cells in vivo (56).

Currently, MTAA-CTL therapy for the treatment of recurrent and refractory solid tumors has begun clinical trials, of which the phase I trial has been completed (ClinicalTrials.gov Identifier: NCT02789228). In this experiment, three kinds of targeted TAAs, WT1, PRAME and survivin, which are specifically expressed or overexpressed in tumor cells, are selected to make MTAACTL attack tumor cells without harming healthy tissue. The recruited patients included 9 Wilms tumor patients (9/18), of whom 7 patients with Wilms tumor were treated with TAA-Ts infusion. In terms of safety, the experiment results showed that all Wilms tumor patients had no doselimited toxicity, no infusion-related adverse events and treatment-related adverse events, so the safety of MTAACTL therapy can be guaranteed. In terms of therapeutic efficacy, of the 15 assessable patients, 11 (73\%) were defined as responders because their condition was stable or improved within 45 days after infusion of TAA-Ts. After initial TAA-Ts treatment, 6 responders still had no progress, with a median of 13.9 months (range, 4.1 to 19.9 months). Patients who received the highest dose showed the best clinical results, with a progression-free survival rate of $73 \%$ at 6 months after TAA-T injection, compared with $38 \%$ at 6 months after prior treatment. The diffusion of antigens and the decrease of tumor-associated antigens in circulation were observed by digital droplet polymerase chain reaction after TAA-T infusion. In this clinical trial, Wilms patients accounted for $50 \%$, so it is reasonable to think that MTAACTL is a promising new treatment for Wilms tumor patients, but the specific effect still needs to be verified by clinical trials specifically for Wilms tumor patients (57).

Immunotherapy is rarely used in Wilms tumor, and the current research is still at the experimentation stage. However, recent research suggests that there is an immunologically involved tumor microenvironment in Wilms tumor, which means that it may be sensitive to immunotherapy (58). This may be the focus of future research in this area.

\section{Conclusions}

In an ideal world where financial resources and time is enough, drug development efforts would be focused on developing pediatric tumor-specific drugs. However, few drugs have been developed specifically for childhood tumors due to a small market for a rare childhood disease. So far, almost all clinical trials opened for Wilms tumor exploit known drugs targeting common pathways which are dysregulated in other adulthood cancers (59). The development of single-cell sequencing technology may provide great convenience for finding this dysregulated pathway. Immunotherapy is similar to targeted therapy. Drug development efforts focused on the development of pediatric solid tumor-specific drugs, rather than Wilms tumor-specific drugs (from https://clinicaltrials. gov/). Compared with targeted therapy in other aspects, the benefits of immunotherapy are more obvious. It not only has the advantages of high precision and low side effects compared with targeted therapy but can also be discontinued during the treatment process without drug dependence (60). At the same time, immunotherapy has less stringent requirements regarding the target (61). At present, CAR-T cell therapy mainly uses the second and third generation of CAR-T cells (43). With the application of the fourth generation CAR-T cells in tumor therapy, it is believed that it will have a better future (62). In the future, targeted therapy and immunotherapy may become the two major adjuvant treatments for postoperative Wilms tumor. 


\section{Acknowledgments}

We thank Yu-Jia Li, for editing the English text of a draft of this manuscript.

Funding: This work was supported by Shanghai Hospital Development Center (grant number, No. SHDC12018X22), The Science Foundation of Shanghai Excellent Youth Scholars (grant number, No. 2017YQ042), The Science Foundation of Shanghai (grant number, No. 17411960600), and Children's National Medical Center (grant number, No. EK112520180301).

\section{Footnote}

Reporting Checklist: The authors have completed the Narrative Review reporting checklist. Available at http:// dx.doi.org/10.21037/tcr-20-3302

Conflicts of Interest: Both authors have completed the ICMJE uniform disclosure form (available at http://dx.doi. org/10.21037/tcr-20-3302). The authors have no conflicts of interest to declare.

Ethical Statement: The authors are accountable for all aspects of the work in ensuring that questions related to the accuracy or integrity of any part of the work are appropriately investigated and resolved.

Open Access Statement: This is an Open Access article distributed in accordance with the Creative Commons Attribution-NonCommercial-NoDerivs 4.0 International License (CC BY-NC-ND 4.0), which permits the noncommercial replication and distribution of the article with the strict proviso that no changes or edits are made and the original work is properly cited (including links to both the formal publication through the relevant DOI and the license). See: https://creativecommons.org/licenses/by-nc-nd/4.0/.

\section{References}

1. Stokes CL, Stokes WA, Kalapurakal JA, et al. Timing of Radiation Therapy in Pediatric Wilms tumor: A Report From the National Cancer Database. Int J Radiat Oncol Biol Phys 2018;101:453-61.

2. Irtan S, Ehrlich PF, Pritchard-Jones K. Wilms tumor: "State-of-the-art" update, 2016. Semin Pediatr Surg 2016;25:250-6.

3. Aldrink JH, Heaton TE, Dasgupta R, et al. Update on
Wilms tumor. J Pediatr Surg 2019;54:390-7.

4. Maschietto M, Piccoli FS, Costa CML, et al. Gene expression analysis of blastemal component reveals genes associated with relapse mechanism in Wilms tumour. Eur J Cancer 2011;47:2715-22.

5. $\mathrm{PDQ}{ }^{\circledR}$ Pediatric Treatment Editorial Board. PDQ Wilms Tumor and Other Childhood Kidney Tumors Treatment [Internet]. Bethesda, MD: National Cancer Institute Press; 2020 [cited 2020 Dec 12]. Available online: https://www. cancer.gov/types/kidney/hp/wilms-treatment-pdq

6. Shukrun R, Shakked NP, Dekel B. Targeted therapy aimed at cancer stem cells: Wilms' tumor as an example. Pediatr Nephrol 2014;29:815-23.

7. Kaneko Y, Okita H, Haruta M, et al. A high incidence of WT1 abnormality in bilateral Wilms tumours in Japan, and the penetrance rates in children with WT1 germline mutation. Br J Cancer 2015;112:1121-33.

8. Hunter RW, Liu Y, Manjunath H, et al. Loss of Dis312 partially phenocopies Perlman syndrome in mice and results in upregulation of Igf2 in nephron progenitor cells. Genes Dev 2018;32:903-8.

9. Chen KS, Stroup EK, Budhipramono A, et al. Mutations in microRNA processing genes in Wilms tumors derepress the IGF2 regulator PLAG1. Genes Dev 2018;32:996-1007.

10. Maschietto M, Charlton J, Perotti D, et al. The IGF signalling pathway in Wilms tumours - A report from the ENCCA Renal Tumours Biology-driven drug development workshop. Oncotarget 2014;5:8014-26.

11. Wagner KD, El Mai M, Ladomery M, et al. Altered VEGF Splicing Isoform Balance in Tumor Endothelium Involves Activation of Splicing Factors Srpk1 and Srsf1 by the Wilms' Tumor Suppressor Wt1. Cells 2019;8:41.

12. Wang J, Fan S, Feng Y, et al. Antiangiogenic therapy for Wilms tumor in an adult and literature review. Anticancer Drugs 2019;30:640-5.

13. Shibuya M. Vascular Endothelial Growth Factor (VEGF) and Its Receptor (VEGFR) Signaling in Angiogenesis: A Crucial Target for Anti- and Pro-Angiogenic Therapies. Genes Cancer 2011;2:1097-105.

14. Interiano RB, McCarville $\mathrm{MB}, \mathrm{Wu} \mathrm{J}$, et al. Pneumothorax as a complication of combination antiangiogenic therapy in children and young adults with refractory/recurrent solid tumors. J Pediatr Surg 2015;50:1484-9.

15. Janku F. Phosphoinositide 3-kinase (PI3K) pathway inhibitors in solid tumors: From laboratory to patients. Cancer Treat Rev 2017;59:93-101.

16. Haddadi N, Lin Y, Travis G, et al. PTEN/PTENP1: 
'Regulating the regulator of RTK-dependent PI3K/Akt signalling', new targets for cancer therapy. Mol Cancer 2018;17:37.

17. Polosukhina D, Love HD, Correa H, et al. Functional KRAS mutations and a potential role for PI3K/AKT activation in Wilms tumors. Mol Oncol 2017;11:405-21.

18. Ludwig N, Werner TV, Backes C, et al. Combining miRNA and mRNA Expression Profiles in Wilms tumor Subtypes. Int J Mol Sci 2016;17:475.

19. Buglyó G, Magyar Z, Gorbe ER, et al. Quantitative RT-PCR-based miRNA profiling of blastemal Wilms' tumors from formalin-fixed paraffin-embedded samples. J Biotechnol 2019;298:11-5.

20. Ludwig N, Nourkami-Tutdibi N, Backes C, et al. Circulating serum miRNAs as potential biomarkers for nephroblastoma. Pediatr Blood Cancer 2015;62:1360-7.

21. Yu X, Li Z, Chan MTV, et al. The roles of microRNAs in Wilms' tumors. Tumour Biol 2016;37:1445-50.

22. Li H, Huang D, Hang S. Salidroside inhibits the growth, migration and invasion of Wilms' tumor cells through down-regulation of miR-891b. Life Sci 2019;222:60-8.

23. Cui M, Liu W, Zhang L, et al. Over-Expression of miR21 and Lower PTEN Levels in Wilms' Tumor with Aggressive Behavior. Tohoku J Exp Med 2017;242:43-52.

24. Liu GL, Yang HJ, Liu B, et al. Effects of MicroRNA$19 \mathrm{~b}$ on the Proliferation, Apoptosis, and Migration of Wilms' Tumor Cells Via the PTEN/PI3K/AKT Signaling Pathway. J Cell Biochem 2017;118:3424-34.

25. Che G, Gao H, Tian J, et al. MicroRNA-483-3p Promotes Proliferation, Migration, and Invasion and Induces Chemoresistance of Wilms' Tumor Cells. Pediatr Dev Pathol 2020;23:144-51.

26. Liu Z, He F, OuYang S, et al. miR-140-5p could suppress tumor proliferation and progression by targeting TGFBRI/SMAD2/3 and IGF-1R/AKT signaling pathways in Wilms' tumor. BMC Cancer 2019;19:405.

27. Wang HF, Zhang YY, Zhuang HW, et al. MicroRNA-613 attenuates the proliferation, migration and invasion of Wilms' tumor via targeting FRS2. Eur Rev Med Pharmacol Sci 2017;21:3360-9.

28. Zhang C, Lv GQ, Cui LF, et al. MicroRNA-572 targets CDH1 to promote metastasis of Wilms' tumor. Eur Rev Med Pharmacol Sci 2019;23:3709-17.

29. Jia W, Deng F, Fu W, et al. Curcumin suppresses wilms' tumor metastasis by inhibiting RECK methylation. Biomed Pharmacother 2019;111:1204-12.

30. Yoshizawa S, Fujiwara K, Sugito K, et al. Pyrroleimidazole polyamide-mediated silencing of KCNQ1OT1 expression induces cell death in Wilms' tumor cells. Int J Oncol 2015;47:115-21.

31. Markovsky E, Vax E, Ben-Shushan D, et al. Wilms tumor NCAM-Expressing Cancer Stem Cells as Potential Therapeutic Target for Polymeric Nanomedicine. Mol Cancer Ther 2017;16:2462-72.

32. Shi B, Li Y, Wang X, et al. Silencing of hypoxia inducible factor-1 by RNA interference inhibits growth of SKNEP-1 Wilms tumour cells invitro, and suppresses tumourigenesis and angiogenesis invivo. Clin Exp Pharmacol Physiol 2016;43:626-33.

33. Zhu S, Fu W, Zhang L, et al. LINC00473 antagonizes the tumour suppressor miR-195 to mediate the pathogenesis of Wilms tumour via IKK. Cell Prolif 2018;51:e12416.

34. Song J, Han J, Liu F, et al. Systematic Analysis of Coronavirus Disease 2019 (COVID-19) Receptor ACE2 in Malignant Tumors: Pan-Cancer Analysis. Front Mol Biosci 2020;7:569414.

35. Giordano G, Campanini N, Donofrio V, et al. Analysis of Cox-2 expression in Wilms' tumor. Pathol Res Pract 2008;204:875-82.

36. Maturu P, Jones D, Ruteshouser EC, et al. Role of Cyclooxygenase-2 Pathway in Creating an Immunosuppressive Microenvironment and in Initiation and Progression of Wilms' Tumor. Neoplasia 2017;19:237-49.

37. Davila ML, Bouhassira DCG, Park JH, et al. Chimeric antigen receptors for the adoptive $T$ cell therapy of hematologic malignancies. Int J Hematol 2014;99:361-71.

38. Jensen TI, Axelgaard E, Bak RO. Therapeutic gene editing in haematological disorders with CRISPR/Cas9. Br J Haematol 2019;185:821-35.

39. Zhao J, Lin Q, Song Y, et al. Universal CARs, universal T cells, and universal CAR T cells. J Hematol Oncol 2018;11:132.

40. Hartmann J, Schuessler-Lenz M, Bondanza A, et al. Clinical development of CAR T cells-challenges and opportunities in translating innovative treatment concepts. EMBO Mol Med 2017;9:1183-97.

41. Petersen CT, Krenciute G. Next Generation CAR T Cells for the Immunotherapy of High-Grade Glioma. Front Oncol 2019;9:69.

42. Abreu TR, Fonseca NA, Gonçalves N, et al. Current challenges and emerging opportunities of CAR-T cell therapies. J Control Release 2020;319:246-61.

43. June CH, Sadelain M. Chimeric Antigen Receptor Therapy. N Engl J Med 2018;379:64-73.

44. Wu W, Zhou Q, Masubuchi T, et al. Multiple Signaling 
Roles of CD3 $\varepsilon$ and Its Application in CAR-T Cell Therapy. Cell 2020;182:855-871.e23.

45. Mohanty R, Chowdhury CR, Arega S, et al. CAR T cell therapy: A new era for cancer treatment. Oncol Rep 2019;42:2183-95.

46. Shah NN, Fry TJ. Mechanisms of resistance to CAR T cell therapy. Nat Rev Clin Oncol 2019;16:372-85.

47. Treger TD, Chowdhury T, Pritchard-Jones K, et al. The genetic changes of Wilms tumour. Nat Rev Nephrol 2019;15:240-51.

48. Casey DL, Cheung NKV. Immunotherapy of Pediatric Solid Tumors: Treatments at a Crossroads, with an Emphasis on Antibodies. Cancer Immunol Res 2020;8:161-6.

49. Buggert M, Vella LA, Nguyen S, et al. The Identity of Human Tissue-Emigrant CD8+ T Cells. Cell 2020;183:1946-1961.e15.

50. Gao W, Ho M. The role of glypican-3 in regulating Wnt in hepatocellular carcinomas. Cancer Rep 2011;1:14-9.

51. Ortiz MV, Roberts SS, Bender JG, et al. Immunotherapeutic Targeting of GPC 3 in Pediatric Solid Embryonal Tumors. Front Oncol 2019;9:108.

52. Shimizu Y, Suzuki T, Yoshikawa T, et al. Next-Generation Cancer Immunotherapy Targeting Glypican-3. Front Oncol 2019;9:248.

53. Ravanpay AC, Gust J, Johnson AJ, et al. EGFR806-CAR T cells selectively target a tumor-restricted EGFR epitope in glioblastoma. Oncotarget 2019;10:7080-95.

54. Wang JX, Dong SJ, Liu QL, et al. The expression and significance of transforming growth factor- $\alpha$, its receptor

Cite this article as: Hong B, Dong R. Research advances in the targeted therapy and immunotherapy of Wilms tumor: a narrative review. Transl Cancer Res 2021;10(3):1559-1567. doi: $10.21037 /$ tcr-20-3302 and proliferation-related antigen $\mathrm{Ki}-67$ in nephroblastoma. Chinese Journal of Urology 2006;27:83-6.

55. Majzner RG, Theruvath JL, Nellan A, et al. CAR T Cells Targeting B7-H3, a Pan-Cancer Antigen, Demonstrate Potent Preclinical Activity Against Pediatric Solid Tumors and Brain Tumors. Clin Cancer Res 2019;25:2560-74.

56. Wang RF, Wang HY. Immune targets and neoantigens for cancer immunotherapy and precision medicine. Cell Res 2017;27:11-37.

57. Hont AB, Cruz CR, Ulrey R, et al. Immunotherapy of Relapsed and Refractory Solid Tumors With Ex Vivo Expanded Multi-Tumor Associated Antigen Specific Cytotoxic T Lymphocytes: A Phase I Study. J Clin Oncol 2019;37:2349-59.

58. Holl EK, Routh JC, Johnston AW, et al. Immune expression in children with Wilms tumor: a pilot study. J Pediatr Urol 2019;15:441.e1-441.e8.

59. Chen C, Garcia HD, Scheer M, et al. Current and Future Treatment Strategies for Rhabdomyosarcoma. Front Oncol 2019;9:1458.

60. Schmidt C. The benefits of immunotherapy combinations. Nature 2017;552:S67-S69.

61. Wang L, Ma N, Okamoto S, et al. Efficient tumor regression by adoptively transferred CEA-specific CAR-T cells associated with symptoms of mild cytokine release syndrome. Oncoimmunology 2016;5:e1211218.

62. Huang $\mathrm{W}$, Liu Y, Hu Y, et al. Construction and function of Glypican-3-targeted fourth-generation chimeric antigen receptor T cells (secreting IL-7 and CCL19). Sheng Wu Gong Cheng Xue Bao 2020;36:979-91. 